\title{
A SVM Approach of Aircraft Conflict Detection in Free Flight
}

\author{
Xu-rui Jiang, ${ }^{1,2}$ Xiang-xi Wen $\mathbb{D}^{1,},{ }^{1,2}$ Ming-gong Wu, ${ }^{1,2}$ Ze-kun Wang, ${ }^{1,2}$ and Xi Qiu ${ }^{3}$ \\ ${ }^{1}$ Air Traffic Control and Navigation College, Air Force Engineering University, Xian 710051, China \\ ${ }^{2}$ National Key Laboratory of Air Traffic Collision Prevention, Xian 710051, China \\ ${ }^{3}$ Nuclear Power Institute of China, Chengdu 610051, China \\ Correspondence should be addressed to Xiang-xi Wen; wxxajy@163.com
}

Received 2 May 2018; Accepted 13 November 2018; Published 6 December 2018

Guest Editor: Ali Tizghadam

Copyright (C) 2018 Xu-rui Jiang et al. This is an open access article distributed under the Creative Commons Attribution License, which permits unrestricted use, distribution, and reproduction in any medium, provided the original work is properly cited.

\begin{abstract}
Probabilistic conflict detection methods typically require high computational burden to deal with complex multiaircraft conflict detection. In this article, aircraft conflict detection is considered as a binary classification problem; therefore, it can be solved by a pattern recognition method. A potential conflict would be identified, as long as its flight data features are extracted and fed to a classifier which has been trained by a large number of flight datasets. Based on this, a new method based on support vector machine (SVM) is employed to detect multiaircraft conflict in "Free Flight" airspace and to estimate the conflict probability. For that purpose, the current positions, velocity vectors, and predicted look-ahead time are selected as detection factors, and the detection model is established by SVM to detect aircraft conflict within look-ahead time during short and medium terms. Moreover, conflict probabilities are determined by the sigmoid function mapping method. Nevertheless, false alarm rate is always a first and foremost problem that troubles air traffic controllers. For the purpose of reducing false alarm rates, Synthetic Minority Over-sampling Technique (SMOTE) method is used to handle imbalanced datasets. Extensive simulation results are presented to illustrate the rationality and accuracy of this method.
\end{abstract}

\section{Introduction}

Nowadays, the demand for air travel continues to grow at a rapid rate with air traffic becoming more and more complex. In this case, collision avoidance (CA), whose task is to maintain a separation between aircraft in air traffic management (ATM), is challenged by increased flight flow. In future, a number of automated decision support technologies, such as Conflict Detection and Resolution (CDR), will be required to enable continued provision of safe and efficient services in increasingly congested skies. Particularly in Free Flight, pilots will have the freedom to choose their trajectory and speed in real time to maximize their flight objectives while maintaining safe separation from neighboring traffic [1], therefore, seeking a fast conflict detection method in Free Flight, which is suitable in both the terminal areas and enroute areas, is extremely significant.

Conflict prediction is based on inexact trajectory prediction $[2,3]$. Deterministic trajectory of an aircraft is influenced by a large number of parameters, particularly due to the wind and also due to the tracking, navigation, and control error, making the trajectory prediction inexact, and random patterns have to be considered. That makes conflict detection become a probability problem, and a number of conflict detection methods based on stochastic process have been proposed. Russel et al. [4] presented a method to estimate mid-term conflict probability of a pair of aircrafts for level flight; in his paper, a coordinate transformation was used to derive an analytical solution. They extended this method to nonlevel flight in [5]. Hu [6] proposed a short-term conflict detection algorithm based on Brownian motion (BM) for level flight. D. Li [7] used J. Hu's [6] algorithm and improved the algorithm to reduce the rate of false alarms. Jilkov et al. [8] proposed a more accurate method for estimating the conflict probability by utilizing the information from multiple model aircraft trajectory prediction. However, previous work on multiaircraft conflict detection in Free Flight is still rare. It is shown in H. Blom's research [9] that the interacting particle system (IPS) algorithm is efficient when more complex air traffic scenarios and more advanced conflict 
zones have to be taken into account. Damien Jacquemart et al. [10] proposed an adaptive algorithm to significantly increase the convergence rate. Moreover, some hierarchical IPS algorithms proposed in research are considered for Free Flight modeling described by advanced stochastic Petri nets [11]. In 2013, Damien Jacquemart [12] also used Markov chain model to simulate aircraft trajectories and applied the important splitting method to estimate conflict probabilities to overcome the flaw of Monte Carlo method which is not efficient to estimate small probabilities. In 2015, Qiao et al. [13] proposed Hidden Markov model-based Trajectory Prediction (HMTP) method to overcome the difficulty of describing the position and behavior of moving objects in a network-constraint environment. Yang et al. [14] proposed a probabilistic reachability analysis approach. In particular, ellipsoidal probabilistic reach sets are determined by formulating a chance-constrained optimization problem and solving it via a simulation-based method called scenario approach. Conflict detection is then performed by verifying if the ellipsoidal reach sets of different aircraft intersect. Their method gives this paper a great inspiration. However, most of the above methods have a common shortcoming, which is every pair of aircraft conflict detection requires a corresponding trajectory prediction.

Actually, the essence of conflict detection is a twoclass classification problem: conflict or nonconflict. In this paper, we introduce SVM to solve the problem of aircraft conflict detection. Support vector machine (SVM) is an excellent two-class classification algorithm, which has been successfully applied in many domains ranging from digit recognition [15] and face recognition [16] to network anomaly detection [17] because of solid theoretical foundation and appealing classification performance. However in air traffic management operation, air traffic controllers (ATCs) expect conflict probability to estimate collision risk and to direct the pilots to avoid the conflicts according to the conflict levels. Standard SVM is a binary classifier: for sample $x$, the output of the SVM, is $+1 /-1$. Some researchers $[18,19]$ modified the SVM outputs into posterior probabilities, and this method is widely applied in many areas $[20,21]$. The calibrated posterior probabilities still inherit the sparseness of the SVM; moreover, they can provide probabilistic prediction decisions. In this paper, we choose Platt's method to evaluate the conflict probability. On these bases, aircraft conflict during short-term to medium-term look-ahead time is predicted, which is suitable for the case of uncertain trajectory prediction error in Free Flight. At the same time, conflict probability can be estimated for ATCs to make a quick decision.

The remainder of the paper is organized as follows. In Section 2, some basic theories used in this paper: the SVM classifier, Platt's probabilistic output, and ellipsoidal protected zone model are introduced. The whole conflict detection process based on SVM is presented. In Section 3, trajectory prediction model based on Brownian motion is introduced to simulate different flight scenarios. Conflict detection experiments on these scenarios are worked out to verify the proposed method. Finally, we conclude in Section 4.

\section{Probabilistic Conflict Detection Model Based on SVM}

2.1. SVM Classifier. Support Vector Machine is a statistical learning method with a good performance [22-27]. Given a dataset $D$ in the form of $\left\{\mathbf{x}_{j}, y_{j}\right\}_{j=1}^{N}$, standard SVM for the binary classification problem maps the feature vector $\mathbf{x} \in R^{d}$ into a high (possibly infinite) dimensional Euclidean space, $H$, using a nonlinear mapping function $\Phi: R^{d} \longrightarrow H$. The goal of support vector machines is to find the optimal separating hyperplane $\mathbf{w} \cdot \Phi(\mathbf{x})+b=0$, which maximizes the margin, and it can be obtained by solving the convex optimization problem:

$$
\begin{array}{ll}
\min _{\mathbf{w}, b, \boldsymbol{\xi}} & \frac{1}{2}\|\mathbf{w}\|^{2}+C\left(\sum_{i=1}^{n} \xi_{i}\right) \\
\text { s.t. } & y_{i}[\mathbf{w} \cdot \Phi(\mathbf{x})+b]-1+\xi_{i} \geq 0 \\
& \xi_{i} \geq 0, i=1, \cdots, n
\end{array}
$$

over $\mathbf{w} \in H, b \in R$, and the nonnegative slack variable $\xi \in R^{N}$. In the above, $C$ is a parameter that balances the size of $\mathbf{w}$ and the sum of $\xi_{i}$. It is well known that the numerical computation of Problem (1) is achieved through its dual formulation. Suppose $\alpha_{i}$ is the Lagrange multiplier corresponding to the $i^{\text {th }}$ inequality, then the dual of (1) can be shown to be

$$
\begin{array}{ll}
\max _{\alpha} & \sum_{i=1}^{n} \alpha_{i}-\frac{1}{2} \sum_{i, j=1}^{n} \alpha_{i} \alpha_{j} y_{i} y_{j} \mathbf{K}\left(\mathbf{x}_{i}, \mathbf{x}_{j}\right) \\
\text { s.t. } & \sum_{i=1}^{n} y_{i} \alpha_{i}=0 \\
& 0 \leq \alpha_{i} \leq C, \quad i=1,2 \cdots, n
\end{array}
$$

where the kernel function $\mathbf{K}\left(\mathbf{x}_{i}, \mathbf{x}_{j}\right)=\Phi\left(\mathbf{x}_{i}\right) \cdot \Phi\left(\mathbf{x}_{j}\right)$ and

$$
\mathbf{w}=\sum_{i=1}^{N} \alpha_{i} y_{i} \Phi\left(\mathbf{x}_{i}\right)
$$

With (3), the expression of the hyperplane $\mathbf{w} \cdot \Phi(\mathbf{x})+b=0$ can be changed into

$$
f(\mathbf{x})=\sum_{i=1}^{N} y_{i} \alpha_{i} \mathbf{K}\left(\mathbf{x}_{i}, \mathbf{x}\right)+b
$$

and serves as the decision function. The predicted class can be defined as

$$
Y=\operatorname{sgn}[f(x)]
$$

The predicted class is +1 if $f(\mathbf{x})>0$ and -1 otherwise.

2.2. Platt's Probabilistic Output. Air traffic controllers (ATCs) expect conflict probability to estimate collision risk; however, standard SVM produces an uncalibrated value that is not a probability. Platt [18] came over this shortcoming and 


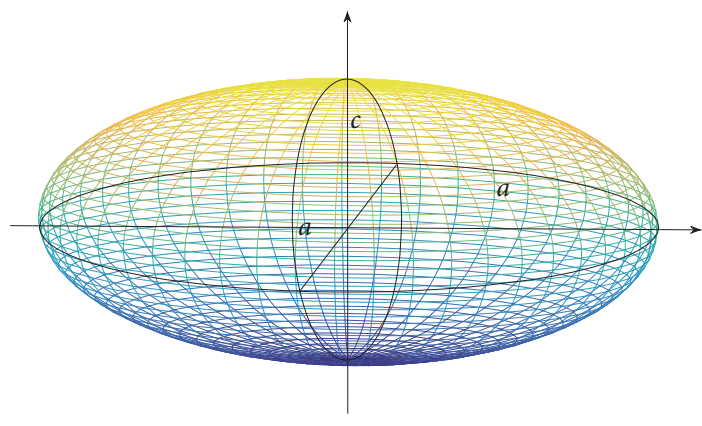

FIGURE 1: A ellipsoidal protected zone.

mapped $f(\mathbf{x})$ into $p(c \mid \mathbf{x})$ through sigmoid function, providing probabilistic information from standard SVM output. Suppose $N_{+}$and $N_{-}$are the numbers of positive $(y=+1)$ and negative $(y=-1)$ samples, respectively, in dataset $D$. The Platt's probability output [28] is

$$
\widehat{p}(c \mid \mathbf{x})=\frac{1}{1+\exp (A f(\mathbf{x})+B)}
$$

where $f(\mathbf{x})$ is the SVM output given by (4) and the parameters $A$ and $B$ are found by minimizing the negative log likelihood of the training data, which is a cross-entropy error function, in the form of

$$
\begin{aligned}
& \min F(A, B) \\
& =\min \left\{-\sum_{i}\left[t_{i} \log \widehat{p}\left(c \mid \mathbf{x}_{i}\right)+\left(1-t_{i}\right) \log \left(1-\widehat{p}\left(c \mid \mathbf{x}_{i}\right)\right)\right]\right\}
\end{aligned}
$$

with $t_{i}=\left(N_{+}+1\right) /\left(N_{+}+2\right)$ if $y_{i}=+1$ and $t_{i}=1 /\left(N_{-}+2\right)$ if $y_{i}=-1$. Hereafter, $\widehat{p}(c \mid \mathbf{x})$ refers to the estimated posterior probability belonging to class +1 given $\mathbf{x}$ obtained from (7), while $\widehat{p}(c \mid \mathbf{x})$ refers to the true but typically unknown posterior probability belonging to class $c$ given $\mathbf{x}$.

2.3. Ellipsoidal Protected Zone Model. The SVM needs training samples to establish the detection model. To identify the potential conflicts, ellipsoidal protected zone is introduced. In Free Flight, aircrafts are surrounded by an imaginary cylindrical volume called the protected zone (PZ). The radius of the PZ is half (2.5 NM) the required horizontal separation standards and the height is equal to the vertical separation $(1000 \mathrm{ft})$. The two aircrafts are said to be in conflict if their PZs overlap. In this paper, an imaginary cylindrical volume is replaced by an ellipsoidal-shaped conflict volume [29, 30], which can be shown in Figure 1.

In this model, we have adopted a slightly modified definition where only the target aircraft is surrounded by the PZ whose dimensions are twice than those defined in the literature [22]. In simplified terms the conflict is identified when potential conflict aircraft enters the PZ of target aircraft within the look-ahead time, and the conflict area is constructed as the following formula:

$$
\frac{\left(x-x_{0}\right)^{2}}{a^{2}}+\frac{\left(y-y_{0}\right)^{2}}{a^{2}}+\frac{\left(z-z_{0}\right)^{2}}{c^{2}} \leq 1
$$

where $\left(x_{0}, y_{0}, z_{0}\right)$ are the coordinates of aircraft located in the ellipsoid center and $(x, y, z)$ are the coordinates of potential conflict aircraft. Set ellipsoidal equatorial radius $a=5 \mathrm{NM}$ and ellipsoidal polar radius $c=2000 \mathrm{ft}$.

The above models are used to judge conflict within lookahead time: in the whole time window from current time $t_{0}$ to look-ahead time $t$. If there is a condition that the distance between a pair of aircraft does not satisfy the protected zone constraint, the conflict occurs. A number of researches have certified that the above model has an effect on actual scenarios during a period of $20 \mathrm{~min}$ because of accumulated error, and this model is applicable for conflict in short-term or medium-term $[6,31]$. Thus, the datasets are selected within $20 \mathrm{~min}$.

2.4. Model Establishment. According to above preliminaries, SVM is adopted to classify conflict conditions into two classes $\{-1,+1\},-1$ refering to "conflict" and +1 to "nonconflict", respectively. A trained C-SVM model is encapsulated as an application module before flight to predict conflict and estimate probability. The flow chart is shown in Figure 2.

Overall, the main process of conflict detection can be divided into three steps:

(1) Data Acquisition. In conflict detection, data acquisition and feature extraction are the fundamental parts. A pair of aircraft position coordinates and velocity vectors are collected: Aircraft A and B's current position coordinates $\overrightarrow{X_{a}}=$ $\left(x_{a}, y_{a}, z_{a}\right), \overrightarrow{X_{b}}=\left(x_{b}, y_{b}, z_{b}\right)$ and Aircraft A and B's velocity vectors $\overrightarrow{v_{a}}=\left(v_{x}, y_{y}, z_{z}\right), \overrightarrow{v_{b}}=\left(v_{x}^{\prime}, v_{y}^{\prime}, v_{z}^{\prime}\right)$. The features are extracted according to the importance of representing flight condition; the features are as follows: the relative distance of the pair of aircraft $\overrightarrow{X r}=\overrightarrow{X_{b}}-\overrightarrow{X_{a}}=\left(x_{r}, y_{r}, z_{r}\right)$, the relative velocity vector $\overrightarrow{V r}=\overrightarrow{v_{b}}-\overrightarrow{v_{a}}=\left(v_{x r}, v_{y r}, v_{z r}\right)$, and predicted look-ahead time $t$. Thus, the feature dimension is reduced to $D=7$ :

$$
\left(x_{r}, y_{r}, z_{r}, v_{x r}, v_{y r}, v_{z r}, t\right)
$$

(2) Data Processing. In actual flight scenario, aircraft conflicts are small probability events, so that the positive samples predominate in collected flight data. In SVM classification problem, when the distribution of the training data among classes is imbalanced, the learning algorithm is generally dominated by the majority classes. The minority classes are normally difficult to be fully recognized. However, conflict samples (negative samples) are quiet important in this problem. In this paper, Synthetic Minority Over-sampling Technique (SMOTE) algorithm $[32,33]$ is applied to handle imbalanced data. The specific approach is to insert virtual negative samples in adjacent negative samples in order to enhance the classification accuracy for the minority classes.

In addition, there are considerable magnitude differences among the 7 indicators. Min-Max Normalization performs a linear transformation on the original data $x$ into the 


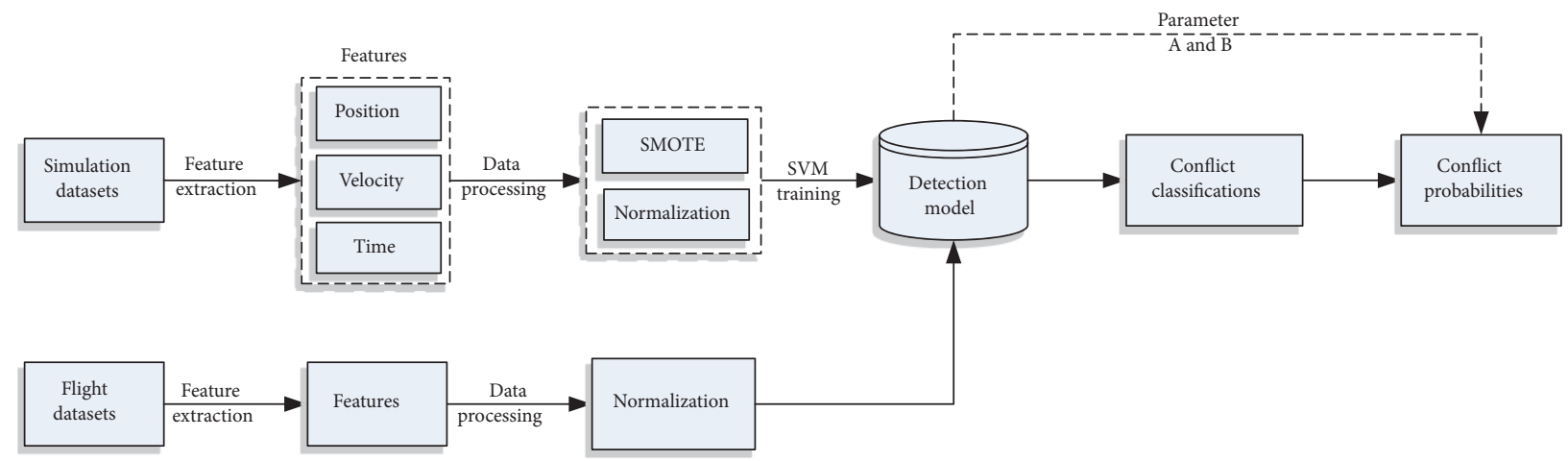

FIgURE 2: Probabilistic conflict detection by SVM flow graph.

specified interval $[-1,+1]$, and the normalization equation is as follows:

$$
Y=\frac{X-X_{\min }}{X_{\max }-X_{\min }}
$$

where $X$ is feature value, $X_{\min }$ and $X_{\max }$ are minimum and maximum values of data, and $Y \in[-1,1]$ is normalized feature value.

(3) Training and Prediction. On the bases of features and labels above, SVM is applied to establish the detection model, in which 1000 samples are trained. Afterwards, parameters $A$ and $B$ are obtained by formula (7). In conflict prediction process, extracted features from flight data are inputted to predict overall conflict in look-ahead time. At the same time, conflict probabilities are estimated by sigmoid function mapping method.

\section{Simulations and Discussion}

To establish and verify the detection model, we need training samples and testing scenarios. In this section, trajectory prediction model based on Brownian motion is introduced to simulate different flight scenarios and acquire training datasets. In training process, 1000 groups of simulation datasets (half positive samples and half negative samples) are used to train the detection model, and actual flight datasets are used for test. Conflict detection experiments in these scenarios are worked out to verify the proposed method.

\subsection{Trajectory Simulation Based on Brownian Motion. Tra-} ditionally, deterministic trajectory of an aircraft is governed by flight mechanic equations. However, a large number of parameters are involved, particularly due to the wind, tracking, navigation, and control error, which makes the trajectory prediction inexact. Here we choose the threedimensional Brownian motion $[6,31]$ as the trajectory prediction model.

A stochastic model is worked out for aircraft trajectories with constant speed following a straight line. Observations presented in [34] show that aircraft position error for a given time is set with Gaussian distribution. The perturbation in position can be treated as a scaled Brownian motion (BM) [6]. One can then assume that

$$
\frac{d X(t)}{d t}=u(t)+\omega(t)
$$

where $X(t)$ is the position of the aircraft at time $t, u(t)$ is a three-dimensional velocity vector, and $\omega(t)$ is random variables following Gaussian distribution with zero mean. Now, if there is an angle $\theta(t)$ at time $t$ between the projecting line of the velocity vector in horizontal plane and $x$-axis, the predicted position is given by

$$
d X(t)=u(t) d t+R(\theta(t)) \sum d B(t)
$$

where $\Sigma$ is the variance growth rate. More precisely, $\Sigma=$ $\operatorname{diag}\left(\sigma_{x}, \sigma_{y}, \sigma_{z}\right)$ models the variance growth rate in the alongtrack, cross-track. and vertical direction. $B(t)$ is a standard three-dimensional Brownian motion, and $R(\theta(t))$ is a rotation matrix:

$$
R(\theta(t))=\left[\begin{array}{ccc}
\cos (\theta(t)) & -\sin (\theta(t)) & 0 \\
\sin (\theta(t)) & \cos (\theta(t)) & 0 \\
0 & 0 & 1
\end{array}\right]
$$

For collecting training and test samples, a pair of aircraft flight scenarios is simulated for 1000 times randomly. Training samples are classified to two classes $\{-1,+1\}$ in look-ahead time $t=\{t \mid t \in(0,20]\}$ by trajectory prediction and protected zone model.

3.2. Validity Analysis. According to the airspace situation, an intersection flying scenario of 10 aircraft has been illustrated. The airspace is established as $90 \mathrm{~km} \times 90 \mathrm{~km}$, and the flight altitude is set from 0 to $15000 \mathrm{~m}$ below. The uncertain trajectories in Free Flight are simulated in Figure 3.

From Figure 3 we can find that the airspace is crowded. The features of 10 aircraft above are extracted as test set, which is inputted to trained classifier to predict conflict during lookahead time $t=3 \mathrm{~min}$, and probability is estimated by formula (5).

To achieve the goal mentioned above, the program LibSVM 3.22 [35] is employed to establish the SVM model. 


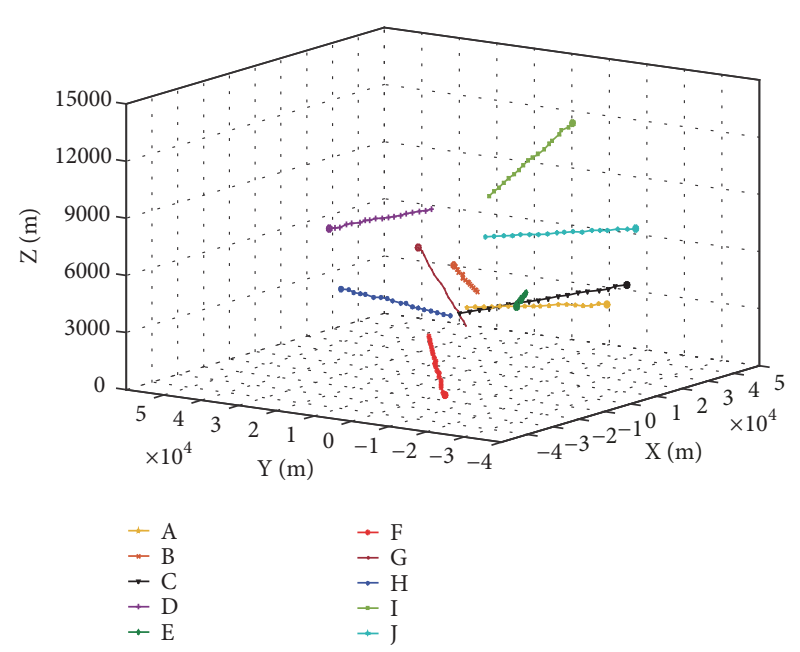

(a)

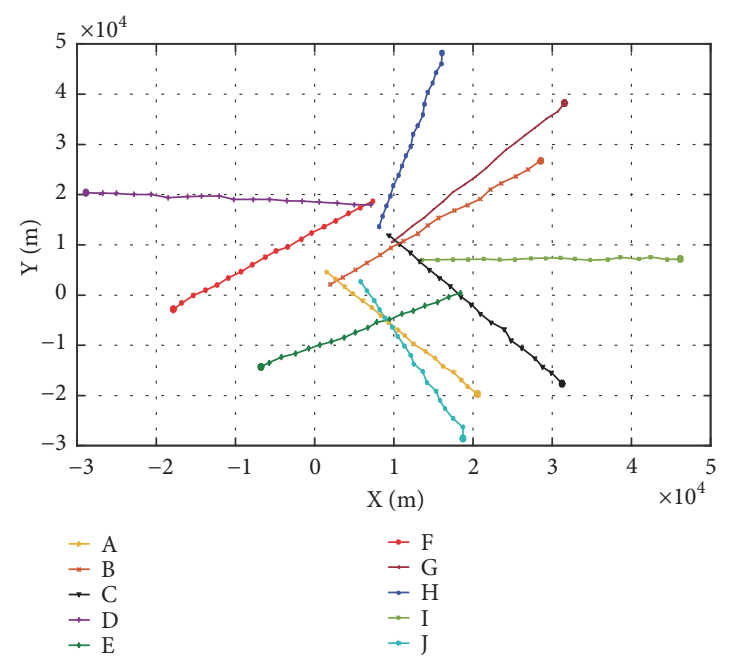

(b)

FIgURE 3: 10 aircraft's flight trajectories: (a) 3D and (b) X-Y axis.

The radial basis function (RBF) kernel was used owing to its nonlinear reflection ability. Grid searches were made on the basis of 5-fold cross-validation to choose a penalty factor $C$ and a kernel parameter $\gamma$. After several searches, a large penalty factor $C=21.3254$ and a kernel parameter $\gamma=0.3515$ were found to handle the problem of overfitting. The parameters $A$ and $B$ are obtained from minimizing the negative log likelihood, which are -1.5881 and -0.0692 , respectively. The result is shown in following matrix $\mathbf{N}_{\mathbf{1 0 \times 1 0}}$ :

$\left.\begin{array}{ccccc}F & G & H & I & J \\ 0.00^{\#} & 6.27 & 2.28 & 0.00^{\#} & 0.00^{\#} \\ 0.00^{\#} & 15.58 & 9.41 & 0.00^{\#} & 0.00^{\#} \\ 0.07 & 84.35 & 9.41 & 0.00^{\#} & 0.00^{\#} \\ 0.00^{\#} & 0.00^{\#} & 0.00^{\#} & 0.25 & 0.00^{\#} \\ 0.00^{\#} & 0.00^{\#} & 0.00^{\#} & 0.00^{\#} & 0.00^{\#} \\ 0 & 65.20 & 60.62 & 0.00^{\#} & 0.00^{\#} \\ & 0 & 52.89 & 0.00^{\#} & 0.00^{\#} \\ & & 0 & 0.00^{\#} & 0.00^{\#} \\ & & & 0 & 0.00^{\#}\end{array}\right)$

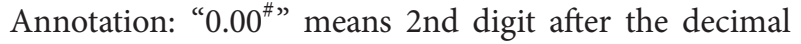
point is less than 5 .

In the upper triangular matrix (14), the elements on the diagonal line are zero. Other elements indicate the conflict probability value of corresponding pair of aircraft. If conflict probability is more than $50 \%$, it is considered that a conflict exists. 10 aircraft conflict detection results in matrix (14) are as follows: there are conflicts between $A$ and $B, A$ and $C, C$ and $G$, $F$ and $G, F$ and $H, G$ and $H$, which are similar to actual conflict detection results in Figure 4. Most conflict probability values are $0.00^{\#}$, which evidences that aircraft conflicts are small probability events. This case reveals the great validity of this method in multiaircraft conflict detection.

3.3. False Alarm Rate and Missing Alarm Rate Analysis. False alarms and missing alarms are vital indicators, which are extremely concerned by air traffic controllers in conflict detection, and they are mainly influenced by the classifier performance. Particularly in this problem, imbalanced datasets have a negative impact on detection results, which have more missed alarms. Therefore, SMOTE algorithm is introduced to improve the classification performance. In this experiment, 


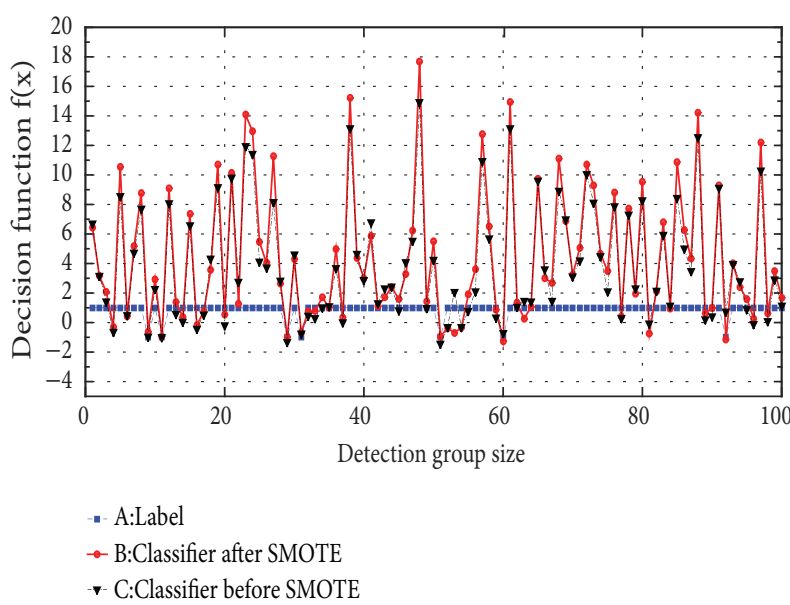

FIGURE 4: Classifier performance comparison before and after SMOTE treatment.

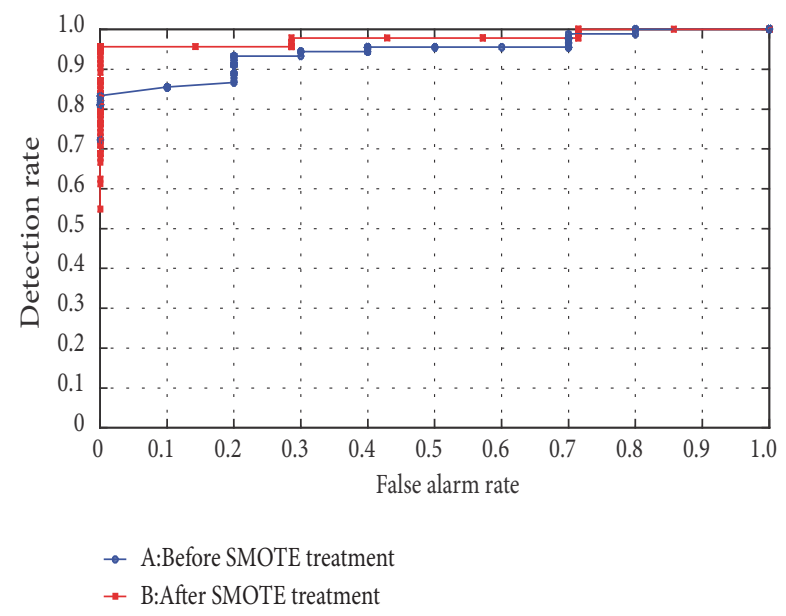

FIGURE 5: ROC curve comparison.

the classification performance whose training datasets are dealt with SMOTE algorithm is compared with that without a treatment. Different scenarios of a pair of aircraft flying are simulated for 100 times, and their samples are collected to test in differently trained classifier, and the conflicts are predicted within $6 \mathrm{~min}$. The results are shown in Figure 4.

In Figure 4, Curve A is class label, -1 refering to "conflict" and +1 to "nonconflict", respectively. Curves B and C are the decision function value of the classifiers. If $f(x)>0$, the test set is classified as positive sample. If $f(x)<0$, the test set is classified as negative sample. Curve $\mathrm{C}$ shows the classification results for samples trained by the classifier before SMOTE treatment: there are 7 conflicts in total which are detected 6 times; in addition, there are 10 time false alarms. The missing alarm rate is $14.29 \%$ and the false alarm rate is $62.50 \%$. Curve B shows the results after SMOTE treatment: every conflict is predicted correctly and $40 \%$ false alarms are rejected effectively. From the results we can know that SMOTE algorithm improves the classification performance to a high level.

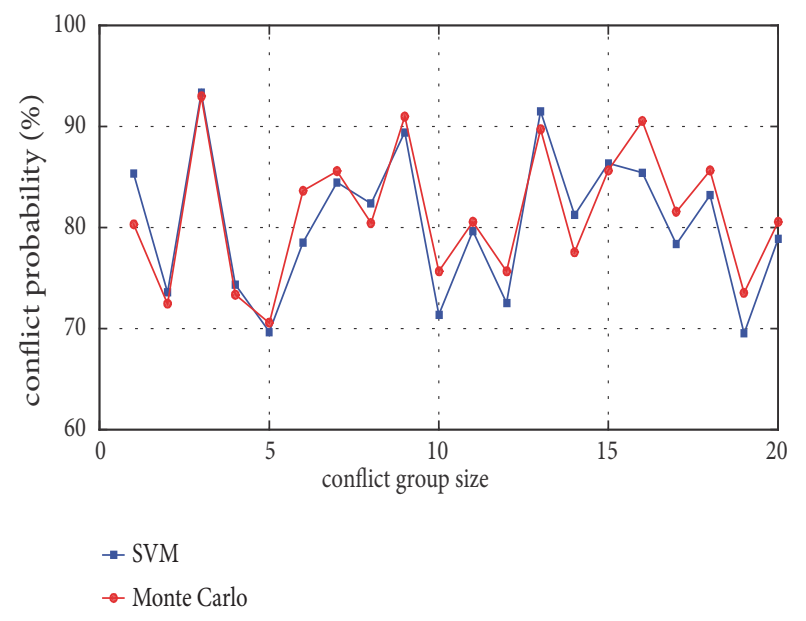

FIGURE 6: Comparison of conflict probability between SVM and Monte Carlo.

Additionally, Receiver Operating Characteristic (ROC) curve is applied to analyze the performance of SMOTE treatment. ROC $[36,37]$ analysis is used for graphical analysis. On the $x$-axis we show the false positive rate (FPR) and on the $y$-axis we show the true positive rate (TPR). ROC curves neatly visualise how the TPR and the FPR change for different (crisp) classifiers or evolve for the same (soft) classifier (or ranker) for a range of thresholds. The notion of threshold is the fundamental idea to adapt a soft classifier to an operating condition. ROC analysis is the tool that illustrates how classifiers and threshold choices perform. Furthermore, it reflects the relationship between the false alarm rate and detection rate. The result is shown in Figure 5.

In Figure 5, Curve A is the ROC curve before SMOTE treatment, and Curve B is plotted after SMOTE treatment. Curve B is upper than Curve A, which shows that classifier trained after SMOTE treatment has a superior performance. When detection rate values are equal, the false alarm rate value on Curve $A$ is greater than the value on Curve $B$, which verifies that the SMOTE algorithm rejects some false alarms effectively.

3.4. Accuracy of Conflict Probability Analysis. The above simulations have shown the conflict detection ability of the proposed method. However, it is undefined whether the conflict probability estimation is effective. In the following research, conflict probabilities estimated by proposed method will be compared with the conflict frequencies simulated by Monte Carlo method in different conflict scenarios for 20 times, and each conflict scenario is simulated by Monte Carlo method for $10^{6}$ times. The comparison result can be shown in Figure 6.

From Figure 6 we can find that the proposed method has a high-fitting-accuracy; conflict probability error of each group sample is less than $10 \%$.

3.5. Stability of Conflict Probability Analysis. In this section, the scenario of a conflict between a pair of aircraft is studied, and the time-dependent change of distances between the 


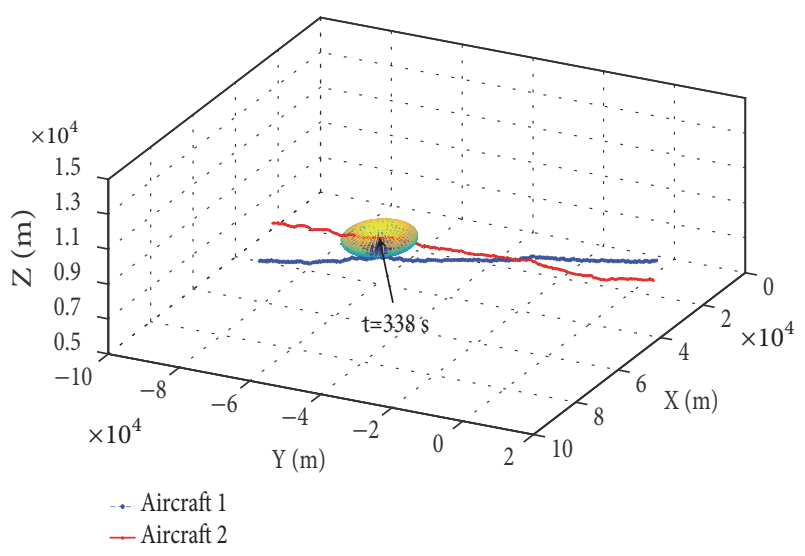

FIgURE 7: A pair of aircraft trajectories.

pair of aircraft and conflict probability within $6 \mathrm{~min}$ is analyzed. Unlike the former analysis, in this section a sample is classified to negative class -1 only if conflict exists at the time $t$. Set time step $T=1 \mathrm{~s}$, and the conflict probability is changing with the relative distance between a pair of aircraft. The different scenarios are as follows. At first, a pair of aircraft departure scenarios are illustrated and discussed: set the heading crossing angle $|\theta| \leq 10^{\circ}$, time interval of departures $t=1 \mathrm{~min}$, and the time period begins upon the last aircraft departure. The trajectories are shown in Figure 7.

The time-dependent change of distances between this pair of aircraft and conflict probability within $6 \mathrm{~min}$ is shown in Figure 8.

In Figure 8, the conflict probability curve exhibits the continuous and linear characteristics, which is identical to actual flight. Since the aircraft has low climb rate in departure, the conflict probabilities remain at a high level (more than $85 \%$ ) in $0-210 \mathrm{~s}$. With the increase of relative distances, the conflict probabilities continue to decrease. At $338 \mathrm{~s}$, the distance is $11752 \mathrm{~m}$, and conflict probability $\mathrm{P}(t=338 \mathrm{~s})=50 \%$. Shortly afterwards, conflicts disappear. With Figure 7 analysis, potential conflict aircraft is at the edge of protected zone at $T=338 \mathrm{~s}$, which verifies the accuracy of the conflict probability output.

Furthermore, three scenarios are illustrated and simulated such as approach to landing phase, intersection flying, and parallel flying in adjacent flight level. In the approach to landing phase, the heading crossing angle is set as $\mid \theta-$ $90^{\circ} \mid \leq 10^{\circ}$. In the intersection flying phase, height difference between aircraft 1 and aircraft 2 is set as $|\Delta h| \leq 600 \mathrm{~m}$, and the heading crossing angle is set as $\left|\theta-90^{\circ}\right| \leq 10^{\circ}$. In the parallel flying phase, set height difference as $|\Delta h| \leq 600 \mathrm{~m}$, the horizontal separation as $|\Delta s-1000 \mathrm{~m}| \leq 4000 \mathrm{~m}$, and the heading crossing angle as $|\theta| \leq 5^{\circ}$. The results of timedependent change of distances between this pair of aircraft and conflict probability within 6 min are shown in Figure 9.

In Figure 9(a), during 0-100 s, the distance between the pair of aircraft is greater than $10 \mathrm{~km}$, and the conflict probability remains at a low level accordingly. After time $t=168 \mathrm{~s}$, since the relative distance is less than $7625 \mathrm{~m}$, the conflict probability is greater than $50 \%$ and a potential

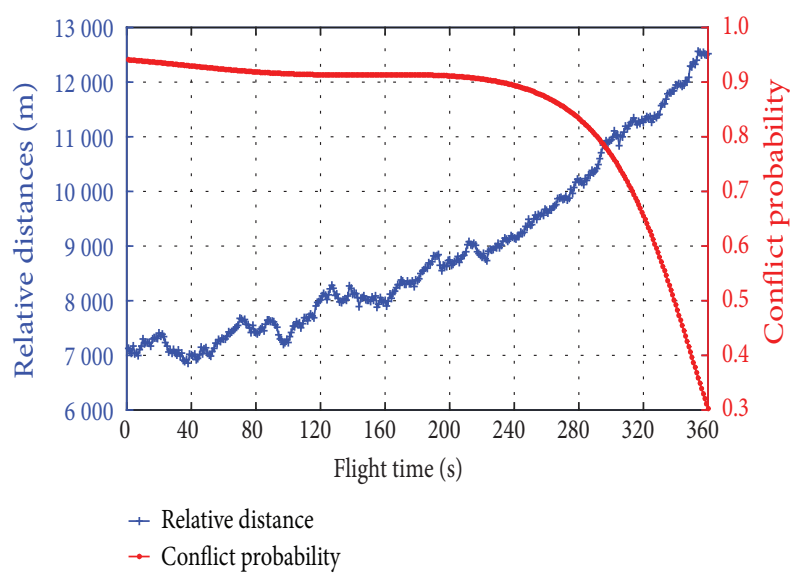

FIGURE 8: Flight time-distances/conflict probability relationship in departure phase.

conflict exists. In Figure 9(b), a conflict exists from the very beginning. Along with the decrease of relative distance, conflict probability continues to increase. With the time period from $210 \mathrm{~s}$ to $240 \mathrm{~s}$, the pair of aircraft flies across each other, and at that time, the relative distance is minimum. The relative distance fluctuates at about $1700 \mathrm{~m}$ because of external factor destabilization such as wind and navigation errors. Conflict probability is about $94 \%$, which remains a high level. After time $t=322 \mathrm{~s}$, the conflict disappears with the increase of the distance. In Figure 9(c), conflict probability ranges from $91 \%$ to $96 \%$ during the whole flying time because of the short distances. Influenced by the wind and the navigation equipment performance, the distances of the pair of aircraft in parallel flight undulate at a narrow range, and the change of conflict probability is opposite to that of the relative distance.

\section{Conclusion}

In this paper, we deal with the conflict detection as a twoclass classification problem. SVM is introduced to establish detection model. Compared with traditional probabilistic conflict detection algorithm, this method avoids setting alarm thresholds artificially which influences the detection accuracy. In addition, the method solves model generalization problem. As long as current positions and velocity vectors of aircraft are obtained, the conflict probabilities between aircraft can be detected quickly. These advantages make it possible that multiaircraft conflicts are detected in real time. The simulation results have shown the high accuracy, stability, and efficiency. Moreover, the proposed algorithm provides a theoretical reference for ATM automation system.

\section{Data Availability}

The data used to support the findings of this study are available from the corresponding author upon request.

\section{Conflicts of Interest}

The authors declare that they have no conflicts of interest. 


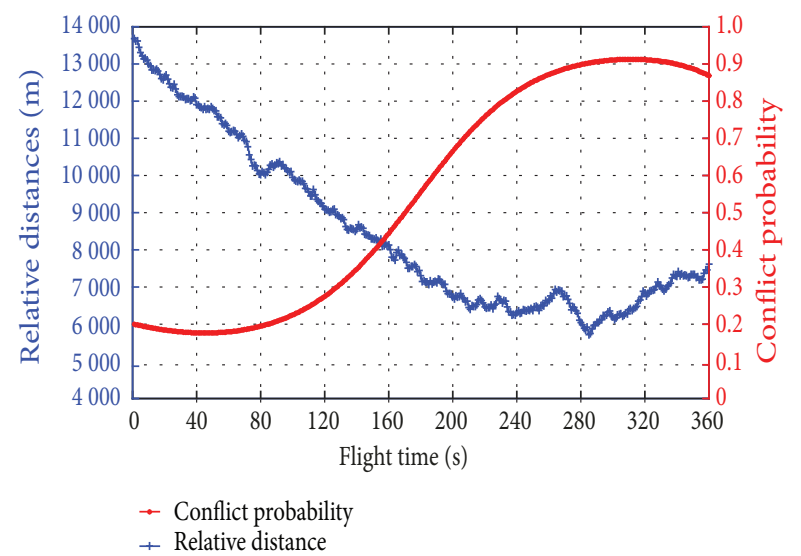

(a)

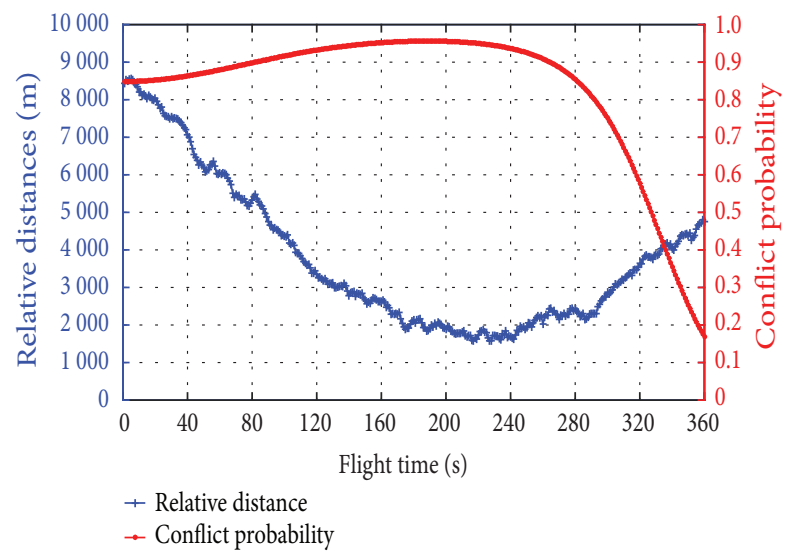

(b)

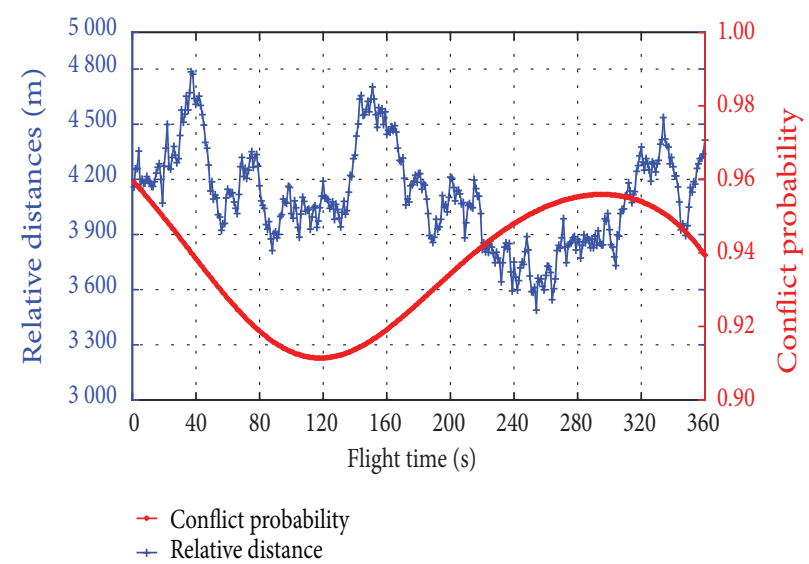

(c)

FIGURE 9: Flight time-distances/conflict probability relationship: (a) in approach to landing phase, (b) in intersection flight, and (c) in parallel flight.

\section{Acknowledgments}

This work is supported by National Natural Science Foundation of China (Grant Nos. 71801221 and 61503409) and Nature Science Basic Research Plan in Shaanxi Province of China (Grant No. 2018JQ7004).

\section{References}

[1] RTCA, "Report of the RTCA Board of Director's Select Committee on Free Flight," Tech. Rep., RTCA Inc, Washington, DC, 1995.

[2] D. P. Thipphavong, C. A. Schultz, A. G. Lee, and S. H. Chan, "Adaptive algorithm to improve trajectory prediction accuracy of climbing aircraft," Journal of Guidance, Control, and Dynamics, vol. 36, no. 1, pp. 15-24, 2013.

[3] S. Hong and K. Lee, "Trajectory prediction for vectored area navigation arrivals," Journal of Aerospace Information Systems, vol. 12, no. 7, pp. 490-512, 2015.

[4] R. A. Paielli and H. Erzberger, "Conflict probability estimation for free flight," Journal of Guidance, Control, and Dynamics, vol. 20, no. 3, pp. 588-596, 1997.

[5] R. A. Paielli and H. Erzberger, "Conflict probability estimation generalized to non-level flight," Air Traffic Control Quarterly, vol. 7, no. 3, pp. 195-222, 1999.
[6] J. Hu, J. Lygeros, M. Prandini, and S. Sastry, "Aircraft conflict prediction and resolution using Brownian motion," in Proceedings of the IEEE Conference on Decision and Control, pp. 24382443, Phoenix, AZ, USA, 1999.

[7] D. Li and D. Cui, "Air traffic control conflict detection algorithm based on Brownian motion," Journal of Tsinghua University, vol. 48 , no. 4 , pp. 477-481, 2008.

[8] V. P. Jilkov, X. R. Li, and J. H. Ledet, "Improved estimation of conflict probability for aircraft collision avoidance," in Proceedings of the 17th International Conference on Information Fusion, pp. 1-7, IEEE Press, Piscataway, NJ, USA, 2014.

[9] H. Blom, G. Bakker, J. Krystul, M. Everdij, B. Klein Obbink, and M. Klompstra, "Sequential Monte Carlo simulation of collision risk in free flight air traffic," Hybridge Report 9, 2005.

[10] D. Jacquemart and J. Morio, "Adaptive interacting particle system algorithm for aircraft conflict probability estimation," Aerospace Science and Technology, vol. 55, pp. 431-438, 2016.

[11] H. A. Blom, J. Krystul, G. J. Bakker, M. B. Klompstra, and B. Klein Obbink, "Free flight collision risk estimation by sequential MC simulation," in Stochastic Hybrid Systems, pp. 248-282, Taylor and Francis group, CRC Press, 2007.

[12] D. Jacquemart and J. Morio, "Conflict probability estimation between aircraft with dynamic importance splitting," Safety Science, vol. 51, no. 1, pp. 94-100, 2013. 
[13] S. Qiao, D. Shen, X. Wang, N. Han, and W. Zhu, "A selfadaptive parameter selection trajectory prediction approach via hidden Markov models," IEEE Transactions on Intelligent Transportation Systems, vol. 16, no. 1, pp. 284-296, 2015.

[14] Y. Yang, J. Zhang, K.-Q. Cai, and M. Prandini, "Multi-aircraft conflict detection and resolution based on probabilistic reach sets," IEEE Transactions on Control Systems Technology, vol. 25, no. 1, pp. 309-316, 2017.

[15] J. M. Saavedra, "Handwritten digit recognition based on pooling SVM-classifiers using orientation and concavity based features," Lecture Notes in Computer Science, vol. 8827, pp. 658-665, 2014.

[16] A. Vijayan, S. Kareem, and J. J. Kizhakkethottam, "Face Recognition Across Gender Transformation Using SVM Classifier," Procedia Technology, vol. 24, pp. 1366-1373, 2016.

[17] S. M. Erfani, S. Rajasegarar, S. Karunasekera, and C. Leckie, "High-dimensional and large-scale anomaly detection using a linear one-class SVM with deep learning," Pattern Recognition, vol. 58, pp. 121-134, 2016.

[18] J. C. Platt, "Probabilistic outputs for support vector machines and comparisons to regularized likelihood methods," in Advances in Large Margin Classifiers, MIT Press, Cambridge, UK, 2000.

[19] A. Madevska-Bogdanova, D. Nikolik, and L. Curfs, "Probabilistic SVM outputs for pattern recognition using analytical geometry," Neurocomputing, vol. 62, no. 1-4, pp. 293-303, 2004.

[20] R. Seifi Majdar and H. Ghassemian, "A probabilistic SVM approach for hyperspectral image classification using spectral and texture features," International Journal of Remote Sensing, vol. 38, no. 15, pp. 4265-4284, 2017.

[21] P. Beigi, R. Rohling, T. Salcudean, V. A. Lessoway, and G. C. Ng, "Detection of an invisible needle in ultrasound using a probabilistic SVM and time-domain features," Ultrasonics, vol. 78, pp. 18-22, 2017.

[22] B. E. Boser, I. M. Guyon, and V. N. Vapnik, "Training algorithm for optimal margin classifiers," in Proceedings of the 5th Annual ACM Workshop on Computational Learning Theory (COLT '92), pp. 144-152, July 1992.

[23] C. Cortes and V. Vapnik, "Support-vector networks," Machine Learning, vol. 20, no. 3, pp. 273-297, 1995.

[24] V. N. Vapnik, The Nature of Statistical Learning Theory, Springer, New York, NY, USA, 1995.

[25] N. Cristianini and J. Shawe-Taylor, An Introduction to Support Vector Machines, Cambridge University Press, Cambridge,UK, 2000.

[26] P. Rebentrost, M. Mohseni, and S. Lloyd, "Quantum support vector machine for big data classification," Physical Review Letters, vol. 113, no. 3, 2014.

[27] Z.-M. Yang, H.-J. Wu, C.-N. Li, and Y.-H. Shao, "Least squares recursive projection twin support vector machine for multiclass classification," International Journal of Machine Learning and Cybernetics, vol. 7, no. 3, pp. 411-426, 2016.

[28] K.-Q. Shen, C.-J. Ong, X.-P. Li, and E. P. V. Wilder-Smith, "Feature selection via sensitivity analysis of SVM probabilistic outputs," Machine Learning, vol. 70, no. 1, pp. 1-20, 2008.

[29] K. Havel and J. Husarčík, "A theory of the tactical conflict prediction of a pair of aircraft," Journal of Navigation, vol. 42, no. 3 , pp. 417-429, 1989.

[30] R. P. Patera, "Space vehicle conflict probability for ellipsoidal conflict volumes," Journal of Guidance, Control, and Dynamics, vol. 30, no. 6, pp. 1818-1822, 2007.
[31] L. Shi and R. Wu, "A probabilistic conflict detection algorithm in terminal area based on three-dimensional Brownian motion," in Proceedings of the 2012 11th International Conference on Signal Processing, ICSP 2012, pp. 2287-2291, China, October 2012.

[32] N. V. Chawla, K. W. Bowyer, L. O. Hall, and W. P. Kegelmeyer, "SMOTE: synthetic minority over-sampling technique," Journal of Artificial Intelligence Research, vol. 16, pp. 321-357, 2002.

[33] L. Torgo, R. P. Ribeiro, B. Pfahringer, and P. Branco, "SMOTE for regression," Lecture Notes in Computer Science, vol. 8154, pp. 378-389, 2013.

[34] R. Paielli and M. Field, "Empirical test of conflict probability estimation," in Proceedings of the USA/Europe Air Traffic Management $R$ and D Seminar, 1998.

[35] C. Chang and C. Lin, "LIBSVM: a Library for support vector machines," ACM Transactions on Intelligent Systems and Technology, vol. 2, no. 3, pp. 75-102, 2011.

[36] J. Hernández-Orallo, "ROC curves for regression," Pattern Recognition, vol. 46, no. 12, pp. 3395-3411, 2013.

[37] J. M. Lampinen, "ROC analyses in eyewitness identification research," Journal of Applied Research in Memory and Cognition, vol. 5, no. 1, pp. 21-33, 2016. 


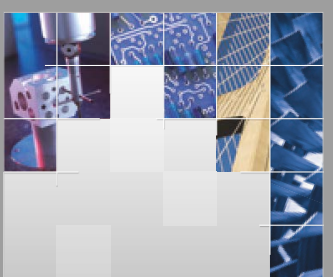

\section{Enfincering}
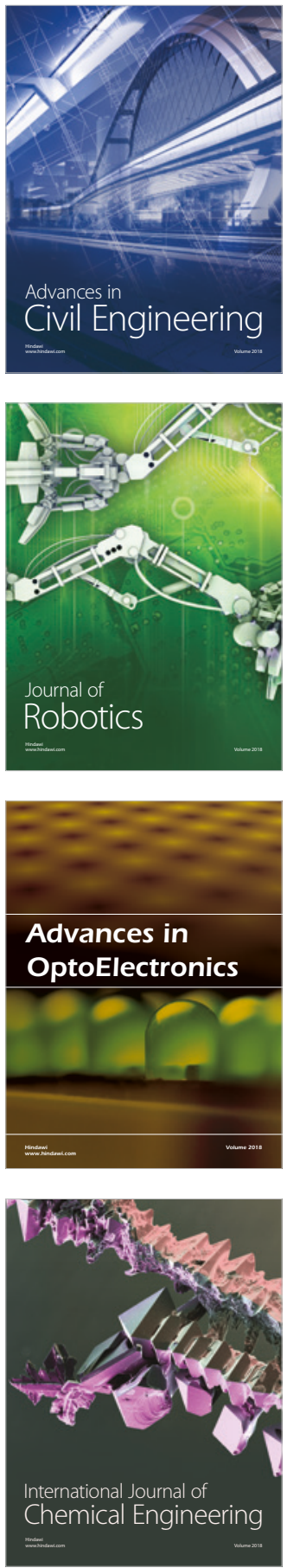

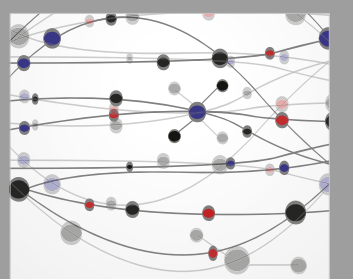

\section{Rotating \\ Machinery}

The Scientific World Journal

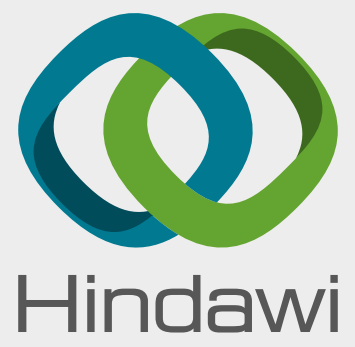

Submit your manuscripts at

www.hindawi.com
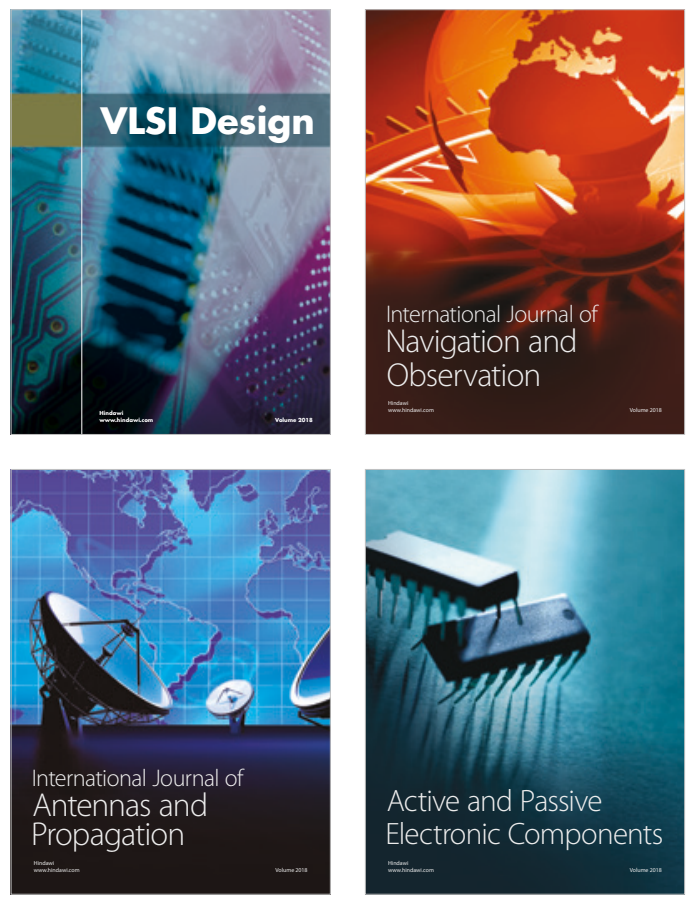
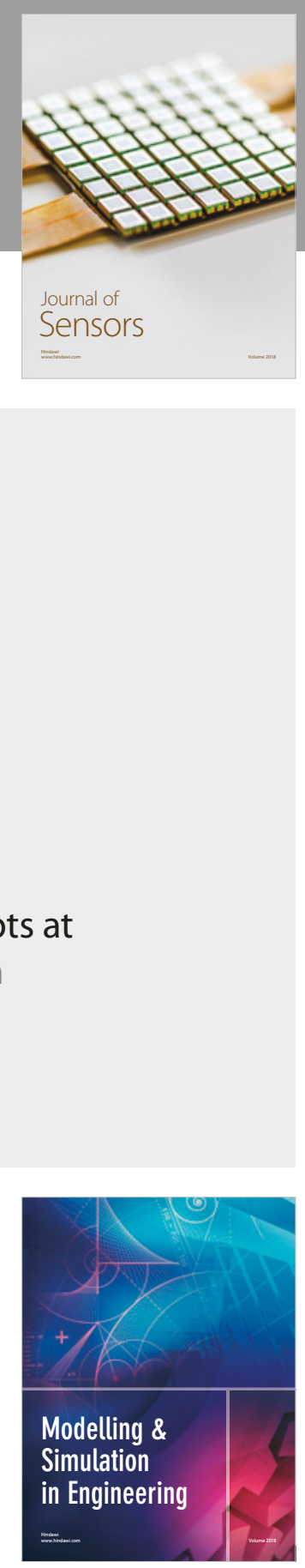

\section{Advances \\ Multimedia}
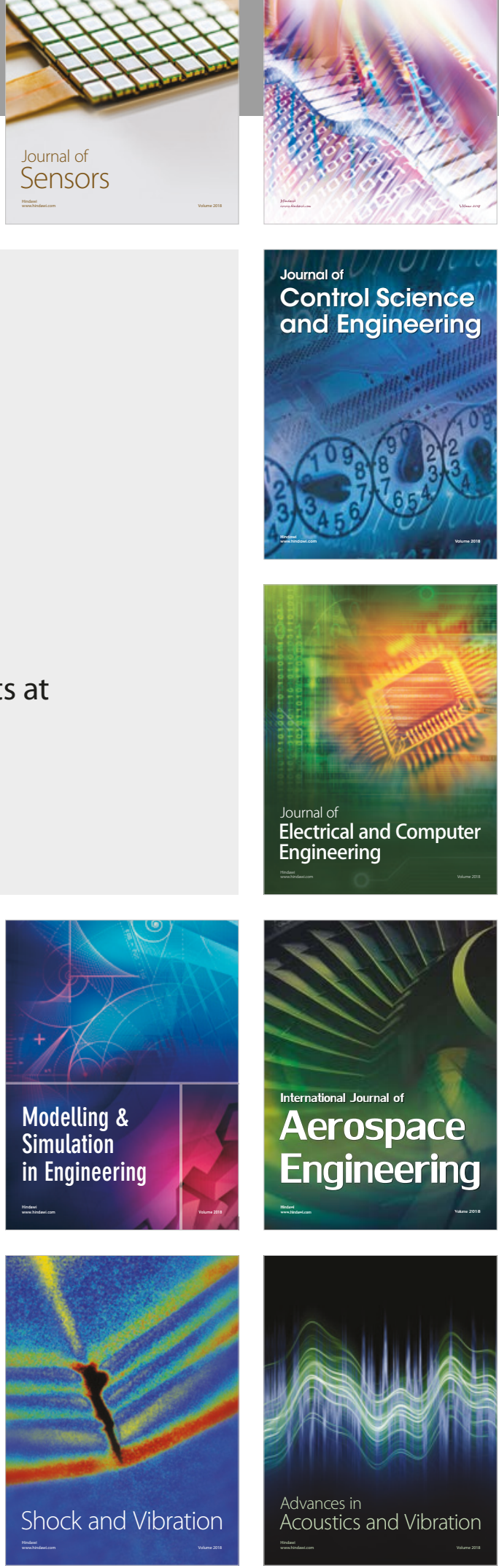